\title{
Plasminogen Activator Inhibitor-1 is Regulated Through Dietary Fat Intake and Heritability: Studies in Twins
}

\author{
Anna Janina Engstler, ${ }^{1,2}$ Turid Frahnow, ${ }^{3,4}$ Michael Kruse, ${ }^{3,5}$ Andreas Friedrich Hermann Pfeiffer, ${ }^{3,5}$ \\ and Ina Bergheim ${ }^{1,2}$ \\ ${ }^{1}$ Institute of Nutrition, SD Model Systems of Molecular Nutrition, Friedrich-Schiller University of Jena, Jena, Germany \\ ${ }^{2}$ Department of Nutritional Sciences, Molecular Nutritional Science, University of Vienna, Vienna, Austria \\ ${ }^{3}$ Department of Clinical Nutrition (KLE), German Institute of Human Nutrition Potsdam-Rehbrücke (DIfE), Nuthetal, \\ Germany \\ ${ }^{4}$ Department of Endocrinology, Diabetes and Nutrition, Charité-Universitätsmedizin Berlin, Berlin, Germany \\ ${ }^{5}$ German Center for Diabetes Research, Nuthetal, Germany
}

\begin{abstract}
In different pathophysiological conditions plasminogen activator inhibitor-1 (PAI-1) plasma concentrations are elevated. As dietary patterns are considered to influence PAI-1 concentration, we aimed to determine active PAl-1 plasma concentrations and mRNA expression in adipose tissue before and after consumption of a high-fat diet (HFD) and the impact of additive genetic effects herein in humans. For 6 weeks, 46 healthy, non-obese pairs of twins (aged 18-70) received a normal nutritionally balanced diet (ND) followed by an isocaloric HFD for 6 weeks. Active PAI-1 plasma levels and PAI-1 mRNA expression in subcutaneous adipose tissue were assessed after the ND and after 1 and 6 weeks of HFD. Active PAl-1 plasma concentrations and PAI-1 mRNA expression in adipose tissue were significantly increased after both 1 and 6 weeks of HFD when compared to concentrations determined after ND $(p<.05)$, with increases of active PAI-1 being independent of gender, age, or changes of BMI and intrahepatic fat content, respectively. However, analysis of covariance suggests that serum insulin concentration significantly affected the increase of active PAI-1 plasma concentrations. Furthermore, the increase of active PAI-1 plasma concentrations after 6 weeks of HFD was highly heritable (47\%). In contrast, changes in PAI-1 mRNA expression in fatty tissue in response to HFD showed no heritability and were independent of all tested covariates. In summary, our data suggest that even an isocaloric exchange of macronutrients - for example, a switch to a fat-rich diet - affects PAI-1 concentrations in humans and that this is highly heritable.
\end{abstract}

Keywords: PAI-1, nutrition, heritability, adipose tissue, insulin resistance, twins

During the last decades, data accumulated suggesting that plasminogen activator inhibitor-1 plasminogen activator inhibitor-1 (PAI-1) might be critical in many disease processes such as cardiovascular and metabolic diseases and even cancer (for overview, see Cesari et al., 2010; De Taeye et al., 2005; Gramling \& Church, 2010; Iwaki et al., 2012). Indeed, it has been shown that PAI1 contributes to the etiology of diseases like thrombosis, fibrosis, and atherosclerosis, not only through altering activities of plasminogen activators and matrix metalloproteinases subsequently affecting fibrinolysis and back-down of extracellular matrix, but also through altering various cell signaling processes (Alessi \& Juhan-Vague, 2006; Flevaris \& Vaughan, 2017; Gramling \& Church, 2010). Indeed, PAI-1 has been shown to alter the activation of hepatocyte growth factor / c-met-dependent signaling cascades through urokinase-type plasminogen activatordependent mechanisms, thereby affecting disease development and progression (Kanuri et al., 2011a; Matsuoka et al., 2006; Sisson et al., 2009). While being expressed in many different cell types (for overview, see Cesari et al., 2010; Devaraj et al., 2003), hepatic and adipose tissue are

RECEIVED 5 March 2017; ACCEPTED 24 May 2017. First published online 21 June 2017.

Address for CORRESPONDENCE: Ina Bergheim, PhD, University of Vienna, Department of Nutritional Sciences, Molecular Nutritional Science, Althanstraße 14, A-1090 Vienna, Austria. E-mail: Ina.bergheim@univie.ac.at 
being discussed to be the key regulating tissues involved in maintaining PAI-1 plasma concentrations (Alessi et al., 1997, 2003; Crandall et al., 1999; Eriksson et al., 1998; Mavri et al., 2001). In plasma, PAI-1 is present in three interconvertible forms: an active conformation, which is stabilized through vitronectin and forms complexes with plasminogen activators thereby resulting in their inhibition, a latent and a substrate form, respectively (for overview, see Yasar Yildiz et al., 2014). For the latter forms, no effects on protease activity have been shown (Yasar Yildiz et al., 2014). Furthermore, expression and plasma concentration of PAI1 are also regulated through various factors like other proinflammatory mediators and endotoxins (for overview, see Cesari et al., 2010), as well as body weight (Appel et al., 2005; Lichtash et al., 2013), and insulin sensitivity (Bastard et al., 2000). In addition, gender (Krishnamurti et al., 1988; Zhang et al., 2014), age (Eren et al., 2014; Krishnamurti et al., 1988), and race/ethnicity (Lutsey et al., 2006) have also been suggested to affect PAI-1 expression and plasma protein concentrations. Results of recent studies further suggest that PAI-1 plasma concentrations are also modulated by additive genetic effects. Indeed, it has been estimated from the results of family and twin studies that 24$71 \%$ of PAI-1 plasma concentrations might be determined through additive genetic effects (Best et al., 2004; Cesari et al., 1999; de Lange et al., 2001; Henry et al., 1998; Hong et al., 1997; Peetz et al., 2004). However, effects of diet and additive genetics on PAI-1 expression in adipose tissue and plasma concentrations have not yet been studied in detail.

Studies in rodents suggest that a diet rich in fat is critical in the development of many metabolic diseases (Amar et al., 2008; Díaz-Rúa et al., 2016; Jacob et al., 2013). Indeed, PAI-1 plasma concentrations were shown to be increased in mice fed a hypercaloric high-fat diet (HFD; Lijnen, 2005; Piao et al., 2016). However, in most of these mouse experiments, HFDs were not only associated with increased PAI-1 concentrations but also increased body weight gain, thereby precluding a clear determination of effects related to diet and/or weight gain/increased adipose tissue mass (Lijnen, 2005; Piao et al., 2016). Furthermore, results of Kalupahana et al. (2011) obtained in mouse feeding studies suggest that alterations of PAI- 1 concentration might be persistent. Indeed, in these studies it was shown that even after normalizing body weight back to that of controls, PAI-1 plasma concentrations of mice exposed to a HFD for 6 months were still significantly higher after body weight was normalized (Kalupahana et al., 2011). In contrast, in human intervention studies, PAI-1 plasma concentrations were not affected by a HFD; however, most studies so far were either rather small (13 subjects) (Marckmann et al., 1992) or short-term (3 days,) and subjects differed in fat intake at study baseline (Ho et al., 1995).

Starting from this background, the aim of the present study was to determine if a short (1 week) or long-term (6 weeks) consumption of an isocaloric HFD has an effect on active PAI- 1 concentrations in plasma and PAI- 1 mRNA expression in adipose tissue of healthy non-obese adults, and whether this effect is dependent on gender, age, or changes of other parameters associated with the intake of a HFD or heritability.

\section{Materials and Methods}

\section{Subjects}

The study was performed in accordance with the ethical principles of the Declaration of Helsinki and good clinical practice. The trial is registered at http://www.clinicaltrials. gov (NCT01631123) and the protocol was approved by the ethics committee of the Charité-University of Medicine, Berlin. All participants signed written informed consent prior to the study. As detailed before (Schüler et al., 2017), a total of 34 monozygotic and 12 dizygotic nonobese twin pairs, aged 18-70 (34 males, 58 females) and a BMI difference between twins of $<3 \mathrm{~kg} / \mathrm{m}^{2}$ were included in the study. Subjects were recruited in Germany in the Berlin-Brandenburg area from a twin register (HealthTwiSt $\mathrm{GmbH}$, Berlin, Germany), through regional newspaper advertisements or information brochures. Criteria for exclusion defined before the study were chronic diseases, drugs affecting the metabolism, pregnancy, or breastfeeding, and a weight change of $>3 \mathrm{~kg} / \mathrm{m}^{2}$ within 3 months prior to the study.

\section{Dietary Intervention}

To standardize participants with regard to fat intake, all study participants were trained to eat a diet according to the recommendation of the German Nutrition Society (DGE; normal diet, ND) as detailed before (Schüler et al., 2017). In brief, absolute energy intake was in accordance to the energy needs of subjects as determined by calculating basal resting energy expenditure through indirect calorimetry, also considering physical activity level, age, and gender. In addition, during the first study phase, energy intake was assessed by 5 -day-dietary protocols and adjusted in case discrepancies were found between initial calculations and results of these protocols. Furthermore, participants were instructed to take in $30 \% \mathrm{E}$ as fat, $55 \% \mathrm{E}$ as carbohydrates, and $15 \% \mathrm{E}$ as protein for the first 6 weeks of the study. Thereafter, dietary intake was changed to an isocaloric HFD consisting of $45 \% \mathrm{E}$ fat, $40 \% \mathrm{E}$ carbohydrates, and 15\% E protein for the next 6 weeks, including two investigation days (one after 1 week of high-fat diet [HFD1] and one after 6 weeks of high-fat diet [HFD6]; also see Figure 1 for study design). One week before tissue und blood samples were collected, participants were asked to consume a standardized diet, of which $70 \%$ of foodstuffs were provided. The remaining time of the study period, participants received a list with 94 food products that they were told to either prefer or avoid during the different dietary intervention periods. During the nutritional intervention phase, 


\begin{tabular}{|c|c|c|c|c|c|c|c|c|c|c|c|c|c|}
\hline & \multicolumn{2}{|c|}{ Normal diet } & \multicolumn{3}{|c|}{$\begin{array}{l}30 \% \mathrm{E} \text { fat } \\
55 \% \mathrm{E} \text { carbohydrate } \\
15 \% \mathrm{E} \text { protein }\end{array}$} & \multirow{2}{*}{$\begin{array}{l}S \\
6\end{array}$} & \multirow{2}{*}{$\frac{S}{7}$} & \multicolumn{2}{|c|}{ High fat diet } & \multicolumn{2}{|c|}{$\begin{array}{l}45 \% \mathrm{E} \text { fat } \\
40 \% \text { E carbohydrate } \\
15 \% \text { E protein }\end{array}$} & \multirow{2}{*}{$\begin{array}{l}\mathrm{S} \\
12 \\
\end{array}$} & \\
\hline & 1 & 2 & 3 & 4 & 5 & & & 8 & 9 & $\begin{array}{l}10 \\
\end{array}$ & 11 & & week \\
\hline I & $\mathbf{N}$ & & $\mathbf{N}$ & & $\mathrm{I}$ & & & $\mathbf{N}$ & & $\mathbf{N}$ & & $\mathbf{N}$ & \\
\hline D & & & & & & & & $\begin{array}{r}\text { HFD1 } \\
\& 8\end{array}$ & & & & $\begin{array}{l}\text { FD6 } \\
888\end{array}$ & 勾 \\
\hline
\end{tabular}

Inital health screen including assessment of anthropometric parameters, a physical examination and anamnesis and an OGTT

$\mathbf{N}$ Nutritional counseling

Blood sample

H Health screen including assessment of anthropometric parameters, magnetic resonance imaging and measurement of fasting insulin and glucose

\& Adipose tissue biopsy

S Standardized diet

\section{FIGURE 1}

Study design. Note: ND = normal diet for 6 weeks; HFD1= high-fat diet for 1 week; HFD6 = high-fat diet for 6 weeks; OGTT = oral glucose tolerance test.

participants were regularly (seven times during the entire study) nutritionally counseled by an experienced dietician as detailed in Figure 1.

\section{Assessment of Health Status}

As detailed by Schüler et al. (2017), general health parameters, including anthropometric measurements and routine blood parameters, were assessed for each participant during an initial health screen before the study began. All participants underwent a physical examination and anamnesis and an oral glucose tolerance test, with blood samples being drawn for the latter $0,30,60,90,120$, and 180 minutes after ingestion of glucose.

After ND, and after 1 and 6 weeks of HFD, respectively, blood, an adipose tissue biopsy and anthropometric data were collected as detailed by Schüler et al. (2017). Furthermore, magnetic resonance imaging to measure the intrahepatic lipid content (IHL) was performed and markers of insulin resistance like serum insulin and glucose concentrations were assessed in fasting blood samples (see Figure 1). Results of these measurements were used to determine homeostatic model assessment of insulin resistance (HOMA-IR); however, due to outlier removal, it was not possible to determine HOMA-IR for all study participants (number of subjects removed for calculation of HOMA-IR: ND: $n=5$, HFD1: $n=3$, HDF6: $n=2$ ). Tissue and blood samples were stored at $-80^{\circ} \mathrm{C}$ until further analysis.

\section{Adipose Tissue Biopsy and Gene Expression Analyses} After local anaesthesia (1\% lidocain) approximately $1 \mathrm{~g}$ of tissue was taken from subcutaneous (s.c.) adipose tissue fat pads, lateral to the umbilicus, using needle aspi- ration. After rinsing briefly with $0.9 \% \mathrm{NaCl}$ saline, the tissue was immediately snap frozen in liquid nitrogen and stored at $-80^{\circ} \mathrm{C}$ for further analyses. Adipose tissue mRNA extraction, cDNA synthesis, and quantitative realtime PCR were performed, as detailed before by Schüler et al. (2017). Primer sequences used were as follows: PAI-1 forward: 5'-CGACATCCTGGAACTGCCCTACC$3^{\prime}$ and reverse: 5'-CACTGTGCCGCTCTCGTTCAC-3', and 60S ribosomal protein L32 (RPL32) forward: 5'CAACGTCAAGGAGCTGGAAGT-3' and reverse: 5'TTGTGAGCGATCTCGGCAC- $3^{\prime}$, the latter serving as a reference gene.

\section{Active PAI-1 and Markers of Insulin Resistance}

Concentrations of functionally active PAI-1 in fasting plasma and serum insulin levels were assessed using commercially available enzyme-linked immunosorbent assay (ELISA) kits (PAI-1: LOXO, Dossenheim, Germany; Insulin: Mercodica AB, Uppsala, Sweden) in accordance with the manufacturer's instructions. For the measurement of glucose concentrations in serum samples, the hexokinase method described by Slein et al. (1950) was used. HOMAIR was calculated as follows: fasting insulin $(\mathrm{mU} / \mathrm{L}) \times$ fasting glucose $(\mathrm{mmol} / \mathrm{L}) / 22.5$.

\section{Statistical Analysis}

Before releasing data for analysis, an analysis of plausibility was performed and unusual values that were outside of 3-fold interquartile range (IQR) were declared as extreme outliers and not considered in further analysis. For anthropometric parameters, no outliers were found. The number of outliers for the other parameters (active PAI-1 plasma 
concentration and PAI-1 mRNA expression in adipose tissue, IHL, fasting glucose, and insulin) was 0-3 depending on time point and variable measured. For the study of heritability, the ACE model was applied as detailed before (Schüler et al., 2017). The ACE model was also checked against the simpler AE-model $(C=0)$, based on a likelihood ratio test and replaced if necessary. Heritability was defined as $A=\sigma_{a^{2}}^{2} / \sigma_{\text {total }}^{2}$ with $\sigma_{\text {total }}^{2}=\sigma_{a^{2}}^{2}+\sigma_{c^{2}}^{2}+\sigma_{e^{2}}^{2}$, and the rest of the variance components were defined the same way with $C$ for the common environment and $E$ for the individual environment. An $A$ value greater than 0.40 was defined as high heritability and used as threshold. Kendall rank correlation coefficient was used to determine correlations between PAI1 mRNA expression in adipose tissue and active PAI- 1 in plasma. To investigate the changes of the parameters, a univariate analysis of variance (ANOVA) with repeated measures and Bonferroni adjustment was used. The requirements for the ANOVA were tested by the Shapiro-Wilk test and Mauchly's sphericity test with ln- and/or GreenhouseGeisser transformation if necessary. Age (as tertiles), sex, as well as the changes of BMI, waist-to-hip ratio (WHR), insulin, HOMA-IR, and IHL were used as covariates in an ANOVA to identify confounding effects on active PAI concentrations and mRNA expression.

Unless otherwise stated, a significance level of $\alpha=0.05$ was used. If the global significance level had to be adjusted due to multiple testing, the Bonferroni method was used. Specifically, when comparing the three different time points, three hypotheses were tested in parallel, resulting in $\alpha_{\text {new }}=0.01 \overline{6}$. However, the alpha was not adjusted by dividing with the number of hypotheses; rather, we adjusted the $p$ value by multiplication. All values are given as median and IQR. The calculations were performed with IBM SPSS Statistics (version 20) and the integrated development environment RStudio (version 0.98.1091), which is based on $\mathrm{R}$ (version 3.1.2). The ACE model was executed in an older version of $\mathrm{R}$ (version 2.1.5), since the necessary package OpenMX was not upward compatible. Graphs were designed with GraphPad Prism (Software version 6, US).

\section{Results}

\section{Characteristics of Study Participants}

Characteristics of participants have in part been published before (Schüler et al., 2017) and are briefly summarized in Table 1. Of the 94 study participants enrolled in the study, 92 completed the intervention and were included in the final analyses. All participants were healthy according to the health assessment, non-obese, with a mean WHR within the normal range as defined by others before (Deutsche Gesellschaft für Sportmedizin und Prävention, 2007). Fasting glucose and insulin concentrations were also within the normal range (Table 1).

Effect of an isocaloric HFD on active PAI-1 plasma concentrations and PAI-1 mRNA expression in adipose tissue as
TABLE 1

Characteristics of Study Participants Before the Study

\begin{tabular}{ll}
\hline & Screening \\
\hline Twin pairs (monozygotic/dizygotic) & $34 / 12$ \\
Gender (female/male) & $58 / 34$ \\
Age (years) & $24.5(21.0 ; 43.0)$ \\
Bodyweight $(\mathrm{kg})$ & $64.1(59.3 ; 76.9)$ \\
BMI $\left(\mathrm{kg} / \mathrm{m}^{2}\right)$ & $22.5(20.9 ; 24.7)$ \\
WHR & $0.80(0.76 ; 0.86)$ \\
Fasting glucose (mmol/L) & $3.98(4.31 ; 4.63)$ \\
Fasting insulin $(\mathrm{mU} / \mathrm{L})$ & $3.83(2.90 ; 5.83)$ \\
HOMA-IR & $0.58(0.71 ; 1.16)$ \\
PAl-1 $(\mathrm{U} / \mathrm{mL})$ & $9.00(3.88 ; 14.50)$
\end{tabular}

Note: Data shown as median with interquartile range in parenthesis. BMI $=$ body mass index; HOMA-IR $=$ homeostatic model assessment of insulin resistance; PAI-1 = plasminogen activator inhibitor-1; WHR = waist-to-hip ratio.

well as metabolic parameters and correlation analysis of active PAI-1 plasma concentrations and PAI-1 mRNA expression in adipose tissue. WHR, fasting glucose concentrations and IHL were not affected by changing the diet of participants to a HFD for 1 week or 6 weeks, respectively; however, both body weight and BMI of participants, as already reported (Schüler et al., 2017), were slightly increased after 6 weeks of consuming a HFD (mean difference: BMI + $0.14 \mathrm{~kg} / \mathrm{m}^{2}$; body weight $\left.+0.40 \mathrm{~kg}, p<.05\right)$. After 1 week of HFD, fasting insulin concentrations and HOMA-IR of study participants were significantly higher when compared to concentrations determined after consuming the ND ( $p$ $<.05$ for both; see also Table 2); however, after 6 weeks of HFD, neither parameters differed from values determined after the ND or 1 week of HFD. Furthermore, after the first week of changing from a ND to HFD, both active PAI-1 concentrations in plasma and PAI-1 mRNA expression in s.c. adipose tissue increased significantly ( $p<.05$ for both). This effect was still found after 6 weeks of HFD $(p<.05$ for both parameters); however, active PAI-1 plasma concentrations and mRNA expression of PAI-1 in s.c. adipose tissue were increased to a similar extent after 1 week and 6 weeks of HFD, respectively, when compared to ND ( $n s$; see Figure 2).

To determine whether mRNA expression of PAI-1 was related to active PAI-1 plasma levels, a correlation analysis was performed. While after 6 weeks of the ND, PAI-1 mRNA concentrations in s.c. adipose tissue and active PAI1 plasma concentrations were not related $(\tau=0.0582 ; p$ $=.4362$ ) a significantly positive correlation of both parameters was found after 1 and 6 weeks of HFD (HFD 1 week: $\tau$ : $=0.2645, p=.0004$; HFD 6 weeks: $\tau=0.2561, p=.0008)$.

Effect of metabolic parameters on active PAI-1 plasma concentrations and PAI-1 mRNA expression in s.c. adipose tissue after changing dietary patterns. Statistical analysis revealed that the increases of PAI-1 activity in plasma and mRNA expression in fatty tissue after 1 and 6 weeks of $\mathrm{HFD}$, respectively, when compared to ND, was independent from covariates like gender and age tertiles (first tertile: 18-22 years, second tertile: $23-30$ years, third tertile: $36-70$ 
TABLE 2

Anthropometric and Metabolic Parameters After the Normal Diet and One Week as Well as Six Weeks of HFD

\begin{tabular}{llll}
\hline & ND & HFD1 & HFD6 \\
\hline Bodyweight $(\mathrm{kg})$ & $64.0(58.6 ; 74.9)^{\mathrm{a}}$ & $63.4(59.1 ; 74.5)^{\mathrm{a}}$ & $64.2(58.6 ; 76.2)^{\mathrm{b}}$ \\
BMI $\left(\mathrm{kg} / \mathrm{m}^{2}\right)$ & $22.2(20.6 ; 24.2)^{\mathrm{a}}$ & $22.1(20.6 ; 24.0)^{\mathrm{a}}$ & $22.5(20.6 ; 24.4)^{\mathrm{b}}$ \\
WHR & $0.80(0.75 ; 0.85)^{\mathrm{a}}$ & $0.80(0.75 ; 0.84)^{\mathrm{a}}$ & $0.79(0.74 ; 0.84)^{\mathrm{a}}$ \\
Fasting glucose (mmol/L) & $5.02(4.68 ; 5.48)^{\mathrm{a}}$ & $5.18(4.77 ; 5.53)^{\mathrm{a}}$ & $5.13(4.75 ; 5.58)^{\mathrm{a}}$ \\
Fasting insulin (mU/L) & $3.81(2.82 ; 5.41)^{\mathrm{a}}$ & $4.67(3.40 ; 6.41)^{\mathrm{b}}$ & $4.54(2.88 ; 5.80)^{\mathrm{a}, \mathrm{b}}$ \\
HOMA-IR & $0.87(0.60 ; 1.28)^{\mathrm{a}}$ & $1.08(0.74 ; 1.47)^{\mathrm{b}}$ & $1.07(0.63 ; 1.36)^{\mathrm{a}, \mathrm{b}}$ \\
IHL (\%) & $0.96(0.56 ; 1.67)^{\mathrm{a}}$ & $0.85(0.57 ; 1.78)^{\mathrm{a}}$ & $0.98(0.53 ; 2.16)^{\mathrm{a}}$ \\
\hline
\end{tabular}

Note: Data shown as median with interquartile range in parenthesis. Values in a row without a common letter differ significantly with $p<.05$ after adjustment for multiple testing (Bonferroni). $\mathrm{BMI}=$ body mass index; HFD1 = high-fat diet for 1 week; HFD6 = high-fat diet for 6 weeks; HOMA-IR = homeostatic model assessment of insulin resistance; IHL = intrahepatic lipid content; ND = normal diet; WHR = waist-to-hip ratio.

A
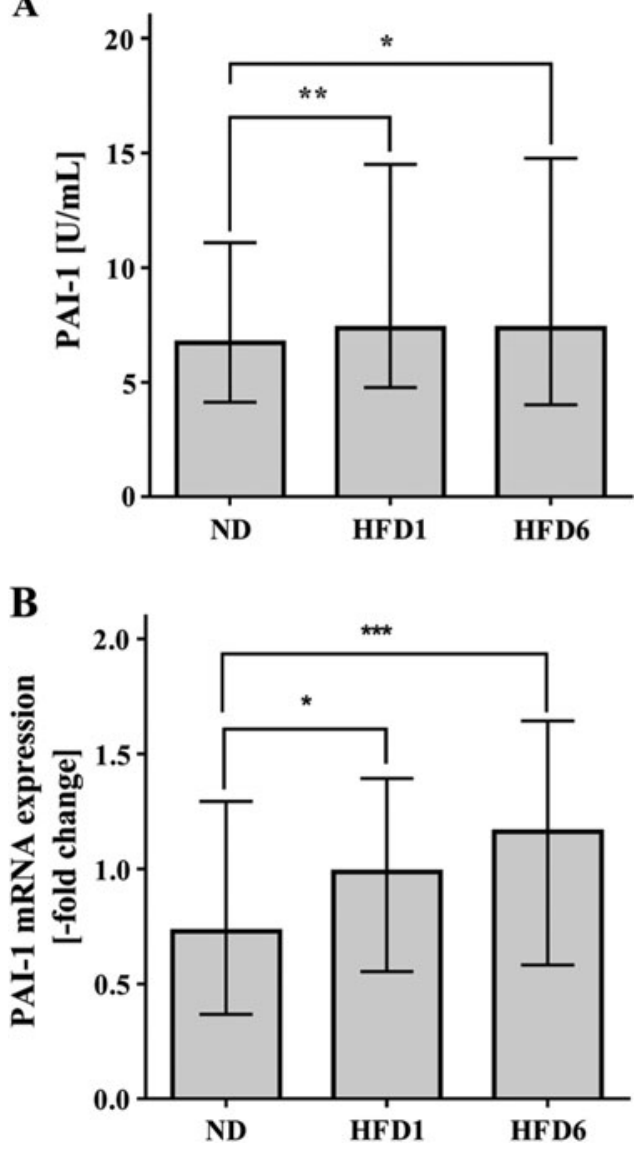

\section{FIGURE 2}

Active PAI-1 concentration in plasma and PAI-1 mRNA expression in adipose tissue after normal and high-fat diet. Note: (A) Active PAI-1 concentration in plasma and (B) PAI-1 mRNA expression in adipose tissue normalized to a housekeeping gene (RPL32). Data are expressed as median and interquartile range. ${ }^{\star} p<.05,{ }^{\star \star} p<$ $.01, \star \star \star p<.001$ after adjustment for multiple testing (Bonferroni). ND = normal diet for 6 weeks; HFD1 = high-fat diet for 1 week; HFD6 = high-fat diet for 6 weeks; PAI-1 = plasminogen activator inhibitor-1.
TABLE 3

Influence of Age, Gender and Metabolic Parameters on Active PAI-1 Plasma Concentrations and PAI-1 mRNA Expression in Adipose Tissue When Participants Changed Dietary Intake From a Normal to a HFD for 1 Week or 6 Weeks

\begin{tabular}{|c|c|c|c|}
\hline & & Active PAl-1 & $\begin{array}{l}\text { PAl-1 mRNA } \\
\text { expression }\end{array}$ \\
\hline \multirow[t]{7}{*}{$\Delta \mathrm{ND} / \mathrm{HFD} 1$} & Gender & ns & ns \\
\hline & Age_tertiles ${ }^{a}$ & ns & ns \\
\hline & $\mathrm{BMI}$ & ns & ns \\
\hline & WHR & ns & ns \\
\hline & Insulin & ** & ns \\
\hline & HOMA-IR & $*$ & ns \\
\hline & $\mathrm{IHL}$ & ns & ns \\
\hline \multirow[t]{7}{*}{$\Delta \mathrm{ND} / \mathrm{HFD} 6$} & Gender & ns & ns \\
\hline & Age_tertiles ${ }^{a}$ & ns & ns \\
\hline & $\mathrm{BMI}$ & ns & ns \\
\hline & WHR & $*$ & ns \\
\hline & Insulin & $*$ & ns \\
\hline & HOMA-IR & ns & ns \\
\hline & $\mathrm{IHL}$ & ns & ns \\
\hline
\end{tabular}

Note: ${ }^{a}$ Age tertiles $=$ first tertile: $18-22$ years, second tertile: $23-30$ years, and third tertile: $36-70$ years. ${ }^{\star} p<.05 ;{ }^{* \star} p<.01 . \mathrm{BMI}=$ body mass index; HOMA-IR = homeostatic model assessment of insulin resistance; ND = normal diet for 6 weeks; HFD1 = high-fat diet for 1 week; HFD6 = high-fat diet for 6 weeks; HFD = high fat diet; IHL = intrahepatic lipid content; $n s=$ not significant; WHR = waist-to-hip ratio.

years), as well as from changes of BMI and IHL. Statistical analysis further revealed that fasting insulin concentration was the only covariate tested that affected the increase of active PAI-1 plasma levels found when participants changed from a ND to HFD (both time points). The change in HOMA-IR was only significantly associated with the increase of active PAI-1 found after 1 week of HFD, while for changes in WHR, a similar association was only found after 6 weeks of HFD. In contrast, changes in PAI-1 mRNA expression in adipose tissue found when subjects changed from the normal diet to the HFD were independent from changes of all parameters analyzed (see Table 3 ).

Effect of heritability on changes of active PAI-1 concentrations in plasma and PAI-1 mRNA expression in s.c. adipose tissue in response to alterations of dietary pattern. To investigate whether the increases found in active PAI-1 concentrations in plasma and PAI-1 mRNA expression in adipose 
A
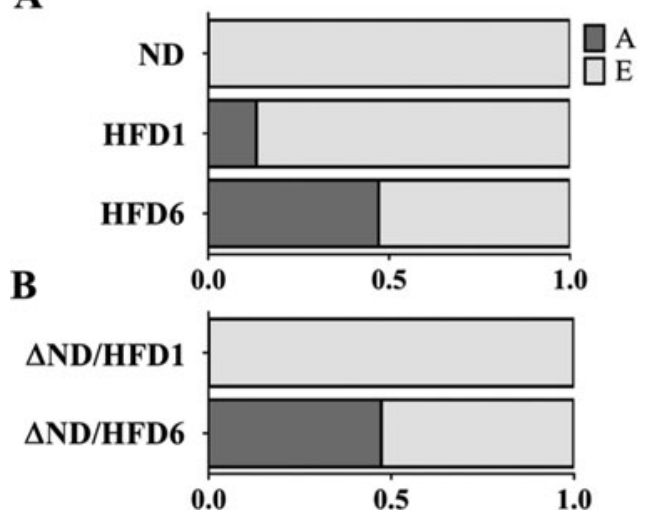

FIGURE 3

Relative distribution of variance determined by additive genetic effects (heritability) and environment of active PAI-1 concentrations in plasma. Note: Distinction of heritability and environmental effects on (A) basal concentrations after normal diet and 1 and 6 weeks of high-fat diet and (B) on the changes of active PAl-1. ACE model: $A=$ additive genetics; $E=$ environment. $N D=$ normal diet for 6 weeks; HFD1 = high-fat diet for 1 week; HFD6 = high-fat diet for 6 weeks.

tissue in response to HFD are inherited, the ACE model was used to distinguish heritability and environmental effects. Active PAI-1 plasma levels showed no heritability $(A=0.0)$ when subjects consumed a ND, whereas when a HFD was eaten heritability estimates increased to $13 \%$ and $47 \%$ after 1 and 6 weeks of HFD, respectively (HFD1, $A=0.134$; HFD6, $A=0.471$; see Figure 3 ). Furthermore, it was determined, if changes of active PAI-1 plasma levels found when subjects changed dietary pattern from the ND to the HFD were heritable. The response to HFD consumed for 1 week showed no heritability, while changes from ND to HFD for 6 weeks showed a heritability of $\sim 47 \%(A=0.473)$. In contrast to plasma concentrations, statistical analysis revealed that PAI-1 mRNA expression in adipose tissue showed no heritability regardless of diet consumed $(A=0.0$ after every investigation day, data not shown). In addition, the changes in PAI-1 mRNA expression from ND to those determined after 1 or 6 weeks of HFD, respectively, were also found not to be heritable $(A=0.0)$.

\section{Discussion}

Results of several studies suggest that increased PAI-1 expression in liver or adipose tissue as well as elevated PAI1 plasma concentrations - be they total or active - are associated with various nutrition-associated diseases (for overview, see Van De Craen et al., 2012). Indeed, it has been shown in mice that knocking down PAI-1 is associated with a marked protection against the development of early phases of non-alcoholic fatty liver disease (NAFLD) but also fibrosis and alcohol-induced steatohepatitis (Bergheim, Guo, Davis, Duveau et al., 2006; Bergheim, Guo, Davis, Lambert et al., 2006; Kanuri et al., 2011a). In these studies, it was shown that PAI-1 is not only involved in the onset of fibrosis but also seems to modulate hepatic lipid export (Kanuri et al., 2011a). Furthermore, weight loss - be it achieved through exercise, energy restriction, or a combination of both - has been reported to be associated with decreased PAI-1 plasma concentrations in humans (Izadpanah et al., 2012; Morel et al., 2011; Österdahl et al., 2008). Results of other studies suggest that PAI-1 concentrations in plasma might already be altered by changes of dietary pattern, such as an increased intake of fiber or total protein (Ampatzoglou et al., 2016; Kashino et al., 2015; Liese et al., 2009; Mortensen et al., 2010). Furthermore, some studies also suggest that $24-71 \%$ of PAI-1 expression and plasma concentration might be regulated through additive genetic effects (Best et al., 2004; Cesari et al., 1999; de Lange et al., 2001; Henry et al., 1998; Hong et al., 1997; Peetz et al., 2004). However, knowledge of the impact of dietary pattern and especially a fat-rich, normo-caloric diet but also heritability in the regulation of PAI-1 is rather limited. In the present study, using a population of monozygotic and dizygotic twins, we aimed to delineate the impact of dietary pattern and heritability on active PAI-1 plasma concentrations and PAI-1 mRNA expression levels in adipose tissue.

While WHR, fasting glucose concentrations, IHL, and body weight as well as BMI were not altered after 1 week of HFD, fasting insulin, HOMA-IR, and active PAI-1 plasma concentrations were significantly increased already after the first week of HFD. This effect of changing dietary pattern on active PAI-1 plasma concentrations and PAI-1 mRNA expression in fatty tissue persisted until the end of the intervention, being accompanied by a slight increase in body weight and BMI but no changes in WHR, fasting glucose or IHL. These findings for active PAI-1 plasma concentrations and PAI-1 mRNA expression in adipose tissue are in line with results of animal studies (Kalupahana et al., 2011; Yan \& DeMars, 2014) and with the study of Masquio et al. (2014) showing a positive association of saturated fatty acid intake and PAI-1 serum concentration in obese adolescents stratified according to their saturated fatty acid intake. However, others found no effects on PAI-1 plasma concentrations in humans under HFD interventions (Ho et al., 1995; Marckmann et al., 1992). Differences between our results and those of others might have resulted from differences in study design (short vs. long-term and standardization period vs. no defined baseline) and ethnicity (Europeans vs. Asians; Ho et al., 1995). Furthermore, results of in vitro studies suggest that PAI-1 mRNA expression in adipose tissue could be regulated through insulin- and triacylglyceroldependent mechanisms (Oller do Nascimento et al., 2009; Sakamoto et al., 2008). However, whether similar molecular mechanisms are also found in humans chronically ingesting a HFD remains to be determined. Also, the slight but significant increase in body weight and BMI after 6 weeks of HFD, which might have resulted from an unintentional change of lifestyle of participants or a metabolic adaptation 
to the changes in dietary pattern, might also have affected active PAI-1 plasma concentrations and PAI-1 mRNA in adipose tissue. Indeed, it has been shown before that an increase in body weight - for example, $+2.6 \mathrm{~kg}$ due to antipsychotic medications - is associated with an increase in PAI-1 plasma concentrations (Fountaine et al., 2010). However, if a markedly lower mean increase in body weight like the one found in the present study $(\sim+0.40 \mathrm{~kg})$ leads to an increase in PAI-1 expression and plasma concentrations remains to be determined. Indeed, when tested as a covariate, no association was found between changes in BMI and alterations of active PAI-1 concentration in plasma or expression in adipose tissue (also see below).

In contrast to the findings for active PAI-1 plasma concentrations and PAI-1 mRNA expression in adipose tissue, after 6 weeks of HFD, fasting insulin levels in serum and HOMA-IR both no longer differed from those found after consumption of the ND, suggesting that subjects might have metabolically adapted to the increased fat intake. Indeed, it has been suggested by the results of animal studies that certain fatty acids - for example, arachidonic acid, $\gamma$ linolenic acid, or linoleic acid - may alter insulin release (Gravena et al., 2002; Lai et al., 2013). It further was shown that a long-term intake of a HFD may be associated with a decrease of this effect (Lai et al., 2013). If similar effects were involved in the present study, and which molecular mechanisms are responsible for the reduction of insulin concentrations and HOMA-IR found when consuming the HFD for an extended period of time, remains to be determined.

Increase of active PAI-1 plasma levels but not PAI-1 $m R N A$ expression in adipose tissue are influenced by insulin levels and HOMA-IR. It has been shown before in animal and human studies that PAI-1 concentrations in plasma are at least in part dependent upon age (for overview, see Eren et al., 2014), gender (Eriksson et al., 2008; Krishnamurti et al., 1988), and body weight (Appel et al., 2005; Lichtash et al., 2013). In the present study, none of the anthropometric and metabolic parameters tested as covariates, with the exception of insulin serum concentrations seemed to have been persistently related to the changes found for active PAI-1 plasma levels after the intake of a HFD. Indeed, it has been discussed that insulin by itself might have an impact on PAI-1 synthesis (for overview, see Alessi \& Juhan-Vague, 2006)) and that PAI-1 might not get 'insulin resistant', but rather responds to insulin even in a situation of insulin resistance (Sartipy \& Loskutoff, 2003). Furthermore, insulin, and even more so insulin resistance, has been shown to be key regulators of PAI-1 expression in hepatic and adipose tissue (Kanuri et al., 2015; Mavri et al., 2001); however, in the present study the response of PAI-1 mRNA expression in adipose tissue to HFD was independent of all tested covariates, including serum insulin and HOMA-IR. This apparent discrepancy might stem from the fact that active PAI-1 levels are not solely regulated at the level of expression. Indeed, changes in external conditions have been proposed to be involved in mediating the conformational change of latent to active PAI-1 (for overview, see Yasar Yildiz et al., 2014). However, in the present study, concentration of active PAI-1 in plasma and PAI-1 mRNA expression in s.c. adipose tissue were positively related after subjects had consumed the HFD, while a similar association was not found after eating the ND. As we only determined active PAI-1 in plasma, it cannot be ruled out that a relation of PAI-1 mRNA expression in s.c. adipose tissue and PAI-1 plasma concentration was missed after the phase subjects had eaten the ND. Also, to date, it has not been fully understood which tissue and cell types are the main contributors to total and active PAI- 1 plasma levels and to which extent the different tissues and cells contribute to plasma levels under different health conditions (e.g., when obesity, insulin resistance, or sepsis is present; Barnard et al., 2016; Bergheim, Luyendyk et al., 2006). Indeed, while studies suggest that PAI-1 mRNA expression in s.c. adipose tissue is related to active PAI-1 plasma levels and fat mass in obese individuals (Alessi et al., 2000; Eriksson et al., 1998), data as to whether this is influenced by the diet consumed by non-obese humans is limited. Therefore, it cannot be ruled out that the lack of relation of PAI-1 mRNA expression in s.c. adipose tissue and active PAI-1 levels in plasma might have resulted from factors consumed with the different diets (e.g., fatty acids). Indeed, results of in vitro studies suggest that PAI-1 mRNA expression in human adipose tissue can be altered depending upon surrounding factors (He et al., 2003).

Taken together, results of the present study further bolster the hypothesis that insulin might be critical in regulating active PAI-1 levels. Furthermore, our results also suggest that fat content in the diet might influence PAI-1 mRNA expression in s.c. adipose tissue and activation of PAI-1 in plasma even in settings of normocaloric intake. However, mechanisms involved remain to be determined.

The late but not the early response of PAI-1 to an isocaloric modification of dietary pattern seems to be heritable. In the present study, active PAI-1 plasma concentrations were not heritable under ND but heritability estimates increased from $13 \%$ after 1 week of HFD to up to $47 \%$ after 6 weeks of HFD. The estimates found after study participants consumed the HFD for 6 weeks are in line with earlier findings of other twin studies (de Lange et al., 2001; Hong et al., 1997; Peetz et al., 2004). Results of these studies also suggest that genetic influences on PAI-1 plasma concentrations are at least in part shared with those for triglycerides (Hong et al., 1997). Indeed, Dichtl et al. (2000) and Nilsson et al. (1999) showed in their studies that very low density lipoprotein (VLDL) through its receptor can modulate PAI-1 expression in endothelial cells and hepatocytes in vitro and in vivo. Similar findings were also reported for oxidized low density lipoprotein (LDL) and lysophosphatidylcholine, and PAI-1 mRNA expression and secretion from endothelial cells (Dichtl et al., 1999). Furthermore, genome-wide 
association studies revealed an association for a common SNP in the peroxisome proliferator-activated receptor gamma gene (rs1801282, also known as Pro12Ala) and PAI1 plasma concentrations (Huang et al., 2012). For Pro12Ala, an association with the risk of metabolic diseases like diabetes type 2 , which is modulated through the dietary fat intake, has been shown (Fisher et al., 2011; Lamri et al., 2012). However, whether alterations of VLDL, or oxidized LDL levels, or a SNP in a gene modulated through fat intake are involved in the lack of a relation to heritability found after the consumption of the ND or 1 week of HFD remains to be determined.

In contrast to the results of active PAI-1 in plasma, induction of PAI-1 mRNA expression in adipose tissue in response to the HFD was not found to be heritable in the present study; however, this might be due to the reasons discussed in detail already above. Taken together, results of the present study suggest that under certain dietary conditions - for example, long-term intake of a HFD - levels of active PAI-1 are regulated at least in part through heritable mechanisms; however, mechanisms involved remain to be determined.

\section{Limitations}

Our study had some limitations that should be taken into consideration when interpreting the data. First, we were only able to enroll a total of 46 pairs of twins, of whom 34 were monozygotic and 12 were dizygotic twins. Second, most participants were young, healthy, and non-obese. Furthermore, all study participants were of European descent. Therefore, it cannot be ruled out that results found in the present study are not representative for older, less healthy (e.g., metabolically abnormal or overweight and obese individuals) individuals, as well as those with a non-European genetic background. Also, in the present study we only examined the effects of an isocaloric exchange of dietary intake from a normal diet to a diet rich in fat. Accordingly, it cannot be ruled out that results might differ in individuals with a general overnutrition that is rich in carbohydrates such as mono- and di-saccharides. Indeed, results of animal studies focusing on monosaccharides suggest that fructose might affect PAI-1 expression in liver (Kanuri et al., 2011b; Spruss et al., 2012). However, whether similar results are also found in humans, and the extent of heritability in carbohydrate- or sugar-induced alterations of PAI-1, remains to be determined. Furthermore, reasons for the slight but significant increase in body weight have not been clarified. It cannot be ruled out that study participants unintentionally changed their lifestyle (e.g., decreased their physical activity or were slightly over-nourished).

\section{Conclusion}

Taken together, our data suggest that even a short-term, but even more so a long-term, intake of a HFD leads to an induction of PAI-1 mRNA expression in adipose tissue and an increase of active PAI-1 concentrations in plasma of non-obese metabolically inconspicuous individuals. Our data further suggests that this HFD-induced increase in active PAI-1 in plasma and PAI-1 mRNA expression in adipose tissue is independent of age, gender, and changes of BMI and IHL, but rather seems to be related at least in part to changes found in insulin serum concentrations. Furthermore, our data also suggest that long-term HFDinduced changes in active PAI-1 plasma concentrations are partially heritable. However, molecular mechanisms underlying these effects remain to be determined.

\section{Financial Support}

This work was supported by the German Federal Ministry of Education and Research (A.P., No. 0315424) and the German Research Foundation (I.B., BE2376/4-2).

\section{Ethical Standards}

The authors assert that all procedures contributing to this work comply with the ethical standards of the relevant national and institutional committees on human experimentation and with the Helsinki Declaration of 1975, as revised in 2008 .

\section{Conflict of Interest}

None.

\section{References}

Alessi, M. C., \& Juhan-Vague, I. (2006). PAI-1 and the metabolic syndrome: Links, causes, and consequences. Arteriosclerosis, Thrombosis, and Vascular Biology, 26, 22002207.

Alessi, M. C., Bastelica, D., Mavri, A., Morange, P., Berthet, B., Grino, M., \& Juhan-Vague, I. (2003). Plasma PAI-1 levels are more strongly related to liver steatosis than to adipose tissue accumulation. Arteriosclerosis, Thrombosis, and Vascular Biology, 23, 1262-1268.

Alessi, M. C., Bastelica, D., Morange, P., Berthet, B., Leduc, I., Verdier, M., ... Juhan-Vague, I. (2000). Plasminogen activator inhibitor 1, transforming growth factor-beta1, and BMI are closely associated in human adipose tissue during morbid obesity. Diabetes, 49, 1374-1380.

Alessi, M. C., Peiretti, F., Morange, P., Henry, M., Nalbone, G., \& Juhan-Vague, I. (1997). Production of plasminogen activator inhibitor 1 by human adipose tissue: Possible link between visceral fat accumulation and vascular disease. $\mathrm{Di}$ abetes, 46, 860-867.

Amar, J., Burcelin, R., Ruidavets, J. B., Cani, P. D., Fauvel, J., Alessi, M. C., ... Ferriéres, J. (2008). Energy intake is associated with endotoxemia in apparently healthy men. The American Journal of Clinical Nutrition, 87, 1219-1223.

Ampatzoglou, A., Williams, C. L., Atwal, K. K., Maidens, C. M., Ross, A. B., Thielecke, F., ... Yaqoob, P. (2016). 
Effects of increased wholegrain consumption on immune and inflammatory markers in healthy low habitual wholegrain consumers. European Journal of Nutrition, 55, 183195.

Appel, S. J., Harrell, J. S., \& Davenport, M. L. (2005). Central obesity, the metabolic syndrome, and plasminogen activator inhibitor-1 in young adults. Journal of the American Academy of Nurse Practitioners, 17, 535-541.

Barnard, S. A., Pieters, M., \& De Lange, Z. (2016). The contribution of different adipose tissue depots to plasma plasminogen activator inhibitor-1 (PAI-1) levels. Blood Reviews, 30, 421-429.

Bastard, J. P., Piéroni, L., \& Hainque, B. (2000). Relationship between plasma plasminogen activator inhibitor 1 and insulin resistance. Diabetes/Metabolism Research and Reviews, 16, 192-201.

Bergheim, I., Guo, L., Davis, M. A., Duveau, I., \& Arteel, G. E. (2006). Critical role of plasminogen activator inhibitor-1 in cholestatic liver injury and fibrosis. The Journal of Pharmacology and Experimental Therapeutics, 316, 592-600.

Bergheim, I., Guo, L., Davis, M. A., Lambert, J. C., Beier, J. I., Duveau, I., ... Arteel, G. E. (2006). Metformin prevents alcohol-induced liver injury in the mouse: Critical role of plasminogen activator inhibitor-1. Gastroenterology, 130, 2099-2112.

Bergheim, I., Luyendyk, J. P., Steele, C., Russell, G. K., Guo, L., Roth, R. A., \& Arteel, G. E. (2006). Metformin prevents endotoxin-induced liver injury after partial hepatectomy. The Journal of Pharmacology and Experimental Therapeutics, 316, 1053-1061.

Best, L. G., North, K. E., Tracy, R. P., Lee, E. T., Howard, B. V., Palmieri, V., \& Maccluer, J. W. (2004). Genetic determination of acute phase reactant levels: The strong heart study. Human Heredity, 58, 112-116.

Cesari, M., Pahor, M., \& Incalzi, R. A. (2010). Plasminogen activator inhibitor-1 (PAI-1): A key factor linking fibrinolysis and age-related subclinical and clinical conditions. Cardiovascular Therapeutics, 28, e72-91.

Cesari, M., Sartori, M. T., Patrassi, G. M., Vettore, S., \& Rossi, G. P. (1999). Determinants of plasma levels of plasminogen activator inhibitor-1: A study of normotensive twins. Arteriosclerosis, Thrombosis, and Vascular Biology, 19, 316320.

Crandall, D. L., Quinet, E. M., Morgan, G. A., Busler, D. E., McHendry-Rinde, B., \& Kral, J. G. (1999). Synthesis and secretion of plasminogen activator inhibitor-1 by human preadipocytes. The Journal of Clinical Endocrinology \& Metabolism, 84, 3222-3227.

de Lange, M., Snieder, H., Ariëns, R. A. S., Spector, T. D., \& Grant, P. J. (2001). The genetics of haemostasis: A twin study. The Lancet, 357(9250), 101-105.

De Taeye, B., Smith, L. H., \& Vaughan, D. E. (2005). Plasminogen activator inhibitor-1: A common denominator in obesity, diabetes and cardiovascular disease. Current Opinion in Pharmacology, 5, 149-154.

Deutsche Gesellschaft für Sportmedizin und Prävention (DGSP). (2007). S 1- Leitlinie Vorsorgeuntersuchung im Sport. Germany.
Devaraj, S., Xu, D. Y., \& Jialal, I. (2003). C-reactive protein increases plasminogen activator inhibitor-1 expression and activity in human aortic endothelial cells: Implications for the metabolic syndrome and atherothrombosis. Circulation, 107, 398-404.

Díaz-Rúa, R., van Schothorst, E. M., Keijer, J., Palou, A., \& Oliver, P. (2016). Isocaloric high-fat feeding directs hepatic metabolism to handling of nutrient imbalance promoting liver fat deposition. International Journal of Obesity, 40, 1250-1259.

Dichtl, W., Ares, M. P., Stollenwerk, M., Giachelli, C. M., Scatena, M., Hamsten, A., ... Nilsson, J. (2000). In vivo stimulation of vascular plasminogen activator inhibitor-1 production by very low-density lipoprotein involves transcription factor binding to a VLDL-responsive element. Thrombosis and Haemostasis, 84, 706-711.

Dichtl, W., Stiko, A., Eriksson, P., Goncalves, I., Calara, F., Banfi, C., ... Nilsson, J. (1999). Oxidized LDL and lysophosphatidylcholine stimulate plasminogen activator inhibitor1 expression in vascular smooth muscle cells. Arteriosclerosis, Thrombosis, and Vascular Biology, 19, 3025-3032.

Eren, M., Boe, A. E., Klyachko, E. A., \& Vaughan, D. E. (2014). Role of plasminogen activator inhibitor-1 in senescence and aging. Seminars in Thrombosis and Hemostasis, 40, 645-651.

Eriksson, M. A., Rask, E., Johnson, O., Carlström, K., Ahrén, B., Eliasson, M., ... Söderberg, S. (2008). Sex-related differences in the associations between hyperleptinemia, insulin resistance and dysfibrinolysis. Blood Coagulation \& Fibrinolysis, 19, 625-632.

Eriksson, P., Reynisdottir, S., Lönnqvist, F., Stemme, V., Hamsten, A., \& Arner, P. (1998). Adipose tissue secretion of plasminogen activator inhibitor-1 in non-obese and obese individuals. Diabetologia, 41, 65-71.

Fisher, E., Schreiber, S., Joost, H. G., Boeing, H., \& Doring, F. (2011). A two-step association study identifies CAV2 rs2270188 single nucleotide polymorphism interaction with fat intake in type 2 diabetes risk. The Journal of $\mathrm{Nu}$ trition, 141, 177-181.

Flevaris, P., \& Vaughan, D. (2017). The role of plasminogen activator inhibitor type-1 in fibrosis. Seminars in Thrombosis and Hemostasis, 43, 169-177.

Fountaine, R. J., Taylor, A. E., Mancuso, J. P., Greenway, F. L., Byerley, L. O., Smith, S. R., ... Fryburg, D. A. (2010). Increased food intake and energy expenditure following administration of olanzapine to healthy men. Obesity 18 , 1646-1651.

Gramling, M. W., \& Church, F. C. (2010). Plasminogen activator inhibitor-1 is an aggregate response factor with pleiotropic effects on cell signaling in vascular disease and the tumor microenvironment. Thrombosis Research, 125, 377-381.

Gravena, C., Mathias, P. C., \& Ashcroft, S. J. (2002). Acute effects of fatty acids on insulin secretion from rat and human islets of Langerhans. Journal of Endocrinology, 173, 73-80.

He, G., Pedersen, S. B., Bruun, J. M., Lihn, A. S., Jensen, P. F., \& Richelsen, B. (2003). Differences in plasminogen activator inhibitor 1 in subcutaneous versus omental adipose tissue 
in non-obese and obese subjects. Hormone and Metabolic Research, 35, 178-182.

Henry, M., Tregouët, D. A., Alessi, M. C., Aillaud, M. F., Visvikis, S., Siest, G., ... Juhan-Vague, I. (1998). Metabolic determinants are much more important than genetic polymorphisms in determining the PAI-1 activity and antigen plasma concentrations: A family study with part of the Stanislas Cohort. Arteriosclerosis, Thrombosis, and Vascular Biology, 18, 84-91.

Ho, C. H., Chwang, L. C., \& Hwang, B. H. (1995). The influence of high fat diet on the fibrinolytic activity. Thrombosis Research, 77, 201-208.

Hong, Y., Pedersen, N. L., Egberg, N., \& de Faire, U. (1997). Moderate genetic influences on plasma levels of plasminogen activator inhibitor- 1 and evidence of genetic and environmental influences shared by plasminogen activator inhibitor-1, triglycerides, and body mass index. Arteriosclerosis, Thrombosis, and Vascular Biology, 17, 27762782.

Huang, J., Sabater-Lleal, M., Asselbergs, F. W., Tregouet, D., Shin, S.-Y., Ding, J., ... Hamsten, A. (2012). Genome-wide association study for circulating levels of PAI-1 provides novel insights into its regulation. Blood, 120, 4873-4881.

Iwaki, T., Urano, T., \& Umemura, K. (2012). PAI-1, progress in understanding the clinical problem and its aetiology. British Journal of Haematology, 157, 291-298.

Izadpanah, A., Barnard, R. J., Almeda, A. J., Baldwin, G. C., Bridges, S. A., Shellman, E. R., ... Roberts, C. K. (2012). A short-term diet and exercise intervention ameliorates inflammation and markers of metabolic health in overweight/obese children. American Journal of Physiology Endocrinology and Metabolism, 303, E542-550.

Jacob, P. S., de Meneses Fujii, T. M., Yamada, M., Borges, M. C., Pantaleão, L. C., Borelli, P., ... Rogero, M. M. (2013). Isocaloric intake of a high-fat diet promotes insulin resistance and inflammation in Wistar rats. Cell Biochemistry and Function, 31(3), 244-253.

Kalupahana, N. S., Voy, B. H., Saxton, A. M., \& MoustaidMoussa, N. (2011). Energy-restricted high-fat diets only partially improve markers of systemic and adipose tissue inflammation. Obesity, 19, 245-254.

Kanuri, G., Ladurner, R., Skibovskaya, J., Spruss, A., Königsrainer, A., Bischoff, S. C., \& Bergheim, I. (2015). Expression of toll-like receptors $1-5$ but not TLR 6-10 is elevated in livers of patients with non-alcoholic fatty liver disease. Liver International, 35, 562-568.

Kanuri, G., Spruss, A., Wagnerberger, S., Bischoff, S. C., \& Bergheim, I. (2011a). Fructose-induced steatosis in mice: Role of plasminogen activator inhibitor-1, microsomal triglyceride transfer protein and NKT cells. Laboratory Investigation, 91, 885-895.

Kanuri, G., Spruss, A., Wagnerberger, S., Bischoff, S. C., \& Bergheim, I. (2011b). Role of tumor necrosis factor alpha (TNFalpha) in the onset of fructose-induced nonalcoholic fatty liver disease in mice. The Journal of Nutritional Biochemistry, 22, 527-534.

Kashino, I., Nanri, A., Kurotani, K., Akter, S., Yasuda, K., Sato, M., ... Mizoue, T. (2015). Association of dietary patterns with serum adipokines among Japanese: A cross-sectional study. Nutrition Journal, 14, 58.

Krishnamurti, C., Tang, D. B., Barr, C. F., \& Alving, B. M. (1988). Plasminogen activator and plasminogen activator inhibitor activities in a reference population. American Journal of Clinical Pathology, 89, 747752.

Lai, M. C., Teng, T. H., \& Yang, C. (2013). The natural PPAR agonist linoleic acid stimulated insulin release in the rat pancreas. The Journal of Veterinary Medical Science, 75, 1449-1454.

Lamri, A., Abi Khalil, C., Jaziri, R., Velho, G., Lantieri, O., Vol, S., ... Fumeron, F. (2012). Dietary fat intake and polymorphisms at the PPARG locus modulate BMI and type 2 diabetes risk in the D.E.S.I.R. prospective study. International Journal of Obesity, 36, 218-224.

Lichtash, C. T., Cui, J., Guo, X., Chen, Y. D., Hsueh, W. A., Rotter, J. I., \& Goodarzi, M. O. (2013). Body adiposity index versus body mass index and other anthropometric traits as correlates of cardiometabolic risk factors. PloS One, 8, e65954.

Liese, A. D., Weis, K. E., Schulz, M., \& Tooze, J. A. (2009). Food intake patterns associated with incident type 2 diabetes: The Insulin Resistance Atherosclerosis Study. Diabetes Care, 32, 263-268.

Lijnen, H. R. (2005). Effect of plasminogen activator inhibitor1 deficiency on nutritionally-induced obesity in mice. Thrombosis and Haemostasis, 93, 816-819.

Lutsey, P. L., Cushman, M., Steffen, L. M., Green, D., Barr, R. G., Herrington, D., ... Folsom, A. R. (2006). Plasma hemostatic factors and endothelial markers in four racial/ethnic groups: The MESA study. Journal of Thrombosis and Haemostasis, 4, 2629-2635.

Marckmann, P., Sandstrom, B., \& Jespersen, J. (1992). Fasting blood coagulation and fibrinolysis of young adults unchanged by reduction in dietary fat content. Arteriosclerosis, Thrombosis, and Vascular Biology, 12, 201-205.

Masquio, D. C., de, P. A., Campos, R. M., Sanches, P. L., Corgosinho, F. C., Carnier, J., ... Dâmaso, A. R. (2014). Saturated fatty acid intake can influence increase in plasminogen activator inhibitor-1 in obese adolescents. Hormone and Metabolic Research, 46, 245-251.

Matsuoka, H., Sisson, T. H., Nishiuma, T., \& Simon, R. H. (2006). Plasminogen-mediated activation and release of hepatocyte growth factor from extracellular matrix. American Journal of Respiratory Cell and Molecular Biology, 35, 705-713.

Mavri, A., Alessi, M. C., Bastelica, D., Geel-Georgelin, O., Fina, F., Sentocnik, J. T., ... Juhan-Vague, I. (2001). Subcutaneous abdominal, but not femoral fat expression of plasminogen activator inhibitor-1 (PAI-1) is related to plasma PAI-1 levels and insulin resistance and decreases after weight loss. Diabetologia, 44, 2025-2031.

Morel, O., Luca, F., Grunebaum, L., Jesel, L., Meyer, N., Desprez, D., ... Goichot, B. (2011). Short-term very lowcalorie diet in obese females improves the haemostatic balance through the reduction of leptin levels, PAI-1 concentrations and a diminished release of platelet and 
leukocyte-derived microparticles. International Journal of Obesity, 35, 1479-1486.

Mortensen, L. S., Thomsen, C., \& Hermansen, K. (2010). Effects of different protein sources on plasminogen inhibitor1 and factor VII coagulant activity added to a fat-rich meal in type 2 diabetes. The Review of Diabetic Studies, 7, 233240.

Nilsson, L., Gåfvels, M., Musakka, L., Ensler, K., Strickland, D. K., Angelin, B., ... Eriksson, P. (1999). VLDL activation of plasminogen activator inhibitor-1 (PAI-1) expression: Involvement of the VLDL receptor. Journal of Lipid Research, 40, 913-919.

Oller do Nascimento, C. M., Ribeiro, E. B., \& Oyama, L. M. (2009). Metabolism and secretory function of white adipose tissue: Effect of dietary fat. Anais da Academia Brasileira de Ciencias, 81, 453-466.

Österdahl, M., Kocturk, T., Koochek, A., \& Wändell, P. E. (2008). Effects of a short-term intervention with a paleolithic diet in healthy volunteers. European Journal of Clinical Nutrition, 62, 682-685.

Peetz, D., Victor, A., Adams, P., Erbes, H., Hafner, G., Lackner, K. J., \& Hoehler, T. (2004). Genetic and environmental influences on the fibrinolytic system: A twin study. Thrombosis and Haemostasis, 92, 344-351.

Piao, L., Jung, I., Huh, J. Y., Miyata, T., \& Ha, H. (2016). A novel plasminogen activator inhibitor-1 inhibitor, TM5441, protects against high-fat diet-induced obesity and adipocyte injury in mice. British Journal of Pharmacology, 173, 26222632.

Sakamoto, K., Sakamoto, T., \& Ogawa, H. (2008). Effects of metabolic risk factors on production of plasminogen activator inhibitor-1 and adiponectin by adipocytes. Circulation Journal, 72, 844-846.

Sartipy, P., \& Loskutoff, D. J. (2003). Expression profiling identifies genes that continue to respond to insulin in adipocytes made insulin-resistant by treatment with tumor necro- sis factor-alpha. The Journal of Biological Chemistry, 278, 52298-52306.

Schüler, R., Osterhoff, M. A., Frahnow, T., Seltmann, A. C., Busjahn, A., Kabisch, S., ... Pfeiffer, A. F. (2017). High-saturated-fat diet increases circulating angiotensinconverting enzyme, which is enhanced by the rs4343 polymorphism defining persons at risk of nutrientdependent increases of blood pressure. Journal of the American Heart Association, 6, e004465.

Sisson, T. H., Nguyen, M. H., Yu, B., Novak, M. L., Simon, R. H., \& Koh, T. J. (2009). Urokinase-type plasminogen activator increases hepatocyte growth factor activity required for skeletal muscle regeneration. Blood, 114, 50525061.

Slein, M. W., Cori, G. T., \& Cori, C. F. (1950). A comparative study of hexokinase from yeast and animal tissues. The Journal of Biological Chemistry, 186, 763-780.

Spruss, A., Kanuri, G., Stahl, C., Bischoff, S. C., \& Bergheim, I. (2012). Metformin protects against the development of fructose-induced steatosis in mice: Role of the intestinal barrier function. Laboratory Investigation, 92, 1020-1032.

Van De Craen, B., Declerck, P. J., \& Gils, A. (2012). The biochemistry, physiology and pathological roles of PAI- 1 and the requirements for PAI-1 inhibition in vivo. Thrombosis Research, 130, 576-585.

Yan, L., \& DeMars, L. C. (2014). Effects of a high-fat diet on spontaneous metastasis of Lewis lung carcinoma in plasminogen activator inhibitor-1 deficient and wild-type mice. PLoS One, 9, e110869.

Yasar Yildiz, S., Kuru, P., Toksoy Oner, E., \& Agirbasli, M. (2014). Functional stability of plasminogen activator inhibitor-1. The Scientific World Journal, Article ID 858293.

Zhang, Y., Zitsman, J. L., Hou, J., Fennoy, I., Guo, K., Feinberg, J., \& Leibel, R. L. (2014). Fat cell size and adipokine expression in relation to gender, depot, and metabolic risk factors in morbidly obese adolescents. Obesity, 22, 691-697. 